\title{
Special Issue on Microfluidics: Theory
}

\section{and Applications}

\author{
Suman Chakraborty ${ }^{1}$, Aloke Kumar ${ }^{2 *}$ and Prosenjit Sen ${ }^{3}$
}

This special issue from the Journal of the Indian Institute of Science is dedicated to the topic of microfluidics, which essentially deals with fundamentals and applications of fluid dynamics over miniaturized (typically, micron or sub-micron) scales. With phenomenal advancements in microfabrication technology over the past decade, rapid developments have taken place in the practical realization of microfluidic systems on a small portable credit card sized device, typically known as lab-on-a-chip or micro-total-analysis-system. Unlike large-scale flows, for flows at these diminutive length scales, physical issues such as interfacial interactions and transport of charged species can become the dominant phenomena. These interactions change the nature of fluid flow significantly and add interesting perspectives to the fundamental governing equations of hydrodynamics. On an application side, it is said that microfluidics is to small-scale flow what the wind tunnel is for large-scale flows. Thus, microfluidic platforms have increasingly being used as mimics to understand flows through complex systems such as porous media and membranes. Finally, the application of microfluidic systems to biomedical applications is widely expected to be the holy grail of research this domain. In particular, the advent of microfluidics is expected to revolutionize the realm of point-of-care diagnostics altogether, especially in the perspectives of their applications in low-cost resource-limited settings.

This special issue brings together six manuscripts belonging to the domain of microfluidics that also elucidate these varied research issues within the discipline. Bandopadhayay and Ghosh discuss the intricacies of interactions between external and induced electric fields and fluid motion. These interactions, which are generally studied under the umbrella of electrokinetics (essentially, electrostatics + hydrodynamics), open up a novel paradigm of actuating pump-free flow in lab-on-a-chip devices. The authors review many interesting facets of electrokinetic flows. Chakraborty and co-workers review fundamental research in the area of flows in micro-confinements with deformable boundaries. The interaction of fluids and walls can have a substantial effect on the underlying fluid physics and the authors discuss multiple coupled phenomena belonging to this realm.

In innovative uses of microfluidics, Debnath and Sadrzadeh discuss how microfluidic platforms can be designed to mimic membranes. Using small microposts separated by a sub-micron distances, microfiltration systems have been successfully mimicked in microfluidic channels. These devices can provide great insights into pore-scale phenomena in membranes, which are otherwise not easily accessible to analytical systems. Williams and co-workers discuss an innovative opto-electrofluidic technology called rapid electrokinetic patterning (REP). REP utilizes optically generated landscapes to drive electrothermal vortices, which can act as a tweezer and manipulate micro/nano-scale particulates. REP is today advancing biomedical research by allowing researchers to detect low-abundance biomarkers for disease diagnosis.

Agrawal and co-workers focus on applications of microfluidic technologies for platelet separation and enrichment. Such technologies allow the development of rapid diagnostic devices with one drop of blood as the test sample. Finally, Toley and co-workers review low-cost paper microfluidic platforms that are enabling a host of assays by simply using microfluidic channels created on paper. These platforms can operate often with zero or very low energy requirements and can be deployed by non-experts.

Overall, these six manuscripts provide a very nice review of the current advancing frontiers of microfluidics. We would like to thank Prof. Guru Row for his support in conceptualizing this special Edition. We would also like to thank the office staff of the Journal, especially Ms. Kavitha Harish, for her outstanding support during the whole process.

\author{
${ }^{1}$ Department \\ of Mechanical \\ Engineering, Indian \\ Institute of Technology \\ Kharagpur, Kharagpur, \\ India. \\ ${ }^{2}$ Department \\ of Mechanical \\ Engineering, Indian \\ Institute of Science, \\ Bangalore, India. \\ ${ }^{3}$ Center for Nano Science \\ and Engineering, Indian \\ Institute of Science, \\ Bangalore, India. \\ *alokekumar@iisc.ac.in
}


Suman Chakraborty is a Professor in the Department of Mechanical Engineering at the Indian Institute of Technology Kharagpur. $\mathrm{He}$ is currently holding the position of Institute Chair Professor. He is also the Dean, Sponsored Research and Industrial Consultancy and Head, School of Medical Science and Technology at the same institute. His research interests include microfluidics and nanofluidics, including their theoretical, computational, and experimental modelling, encompassing the underlying fundamentals as well as biomedical, and energy-related, environmental, and other applications. Substantial proportions of his current research projects pertain to bio-microfluidics with focus on technologies for affordable healthcare. He has been the recipient of many prestigious awards and fellowships, including the Santi Swarup Bhatnagar Prize, fellowships of all the relevant National Academies of Science and Engineering as well some leading International bodies such as the American Society of Mechanical Engineering, American Physical Society, and Royal Society of Chemistry (UK).

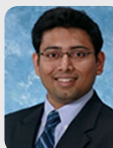

Dr. Aloke Kumar received his Bachelors and Masters degrees from the Indian Institute of Technology Kharagpur, India, in 2005 and his Ph.D. in Mechanical Engineering from Purdue
University, West Lafayette, USA, in 2010. His doctoral work is one of the founding works in the area of opto-electrofluidics-an emerging area of microfluidics, which today is benefitting applications from colloidal physics to biomedical diagnostics. Dr. Kumar joined University of Alberta's Mechanical Engineering Department in 2013, where he was the Canada Research Chair in Microfluidics for Biological Systems. He moved to the Indian Institute of Science in 2017, where he re-established his Soft Matter Lab with a focus on bacterial biofilms and polymeric flows.

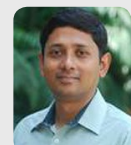

Prosenjit Sen was born in Calcutta, India, in 1978. He received the B.Tech. degree in manufacturing science and engineering from Indian Institute of Technology Kharagpur, India, in 2000, and the Ph.D. degree in mechanical engineering from the University of California, Los Angeles, in 2007. He is currently with the Centre for Nano Science and Engineering, Indian Institute of Science, Bangalore. His research interests include microfluidic devices, fluids as interfaces, electrowetting-on-dielectric (EWOD) devices and $3 \mathrm{D}$ integration of heterogeneous devices. 